\title{
Violência contra a pessoa idosa no contexto português: questões e contradições
}

\author{
Maria Júlia Carneiro Fernandes*, Alcione Leite da Silva**
}

\section{Resumo}

A violência contra a pessoa idosa é um fenômeno universal com vários cenários como pano de fundo. Num universo de 53 países europeus, Portugal destaca-se com um dos maiores índices (39,4\%). Considerando a sua elevada subnotificação, os dados epidemiológicos constituem apenas a ponta do iceberg de uma cultura relacional de dominação, de conflitos intergeracionais ou de negligências familiares ou institucionais. Este artigo tem como objetivo dar visibilidade ao fenômeno da violência contra a pessoa idosa em Portugal considerando as suas múltiplas formas de expressão e fatores predisponentes no atual processo de envelhecimento mundial da população. Neste sentido, foi realizada uma revisão narrativa para subsidiar esta reflexão no contexto português, tendo como contraponto os avanços alcançados em outros países. Conclui-se que o reconhecimento deste tipo de violência como um problema sério em Portugal requer uma consciência social integradora e um esforço de complementaridade das diversas áreas do conhecimento. Apenas desta forma será possível salvaguardar os direitos da pessoa idosa e encontrar respostas eficazes e humanizadas que permitam prevenir ou atenuar os efeitos da violência na sociedade.

Palavras-chave: Pessoa idosa. Saúde pública. Violência.

\section{Introdução}

$\mathrm{Na}$ sociedade contemporânea, a longevidade humana representa uma grande conquista histórica e social, mas também um problema pelas consequências multidimensionais que encerra. As transformações sociais, culturais, políticas e econômicas, advindas desse fenômeno, impedem que o mesmo se torne um assunto pacífico. Portugal tem revelado dificuldades em responder eficazmente a este apelo. Na última década, o número de pessoas idosas com mais de 65 anos cresceu para 2.023 milhões, representando cerca de $19 \%$ da população total comparativamente com os

* Enfermeira graduada em Cuidados Intensivos. Mestre em Gerontologia. Doutoranda em Geriatria \& Gerontologia. Endereço para correspondência: Rua dos Bacalhoeiros, n. 12, 3800-905 S. Jacinto, Aveiro, Portugal. E-mail: juliafernandes@sapo.pt

** Enfermeira. Doutora em Filosofia em Enfermagem. Professora associada convidada do Departamento de Saúde da Universidade de Aveiro. E-mail: alciones06@gmail.com

$\rightarrow$ http://dx.doi.org/10.5335/rbceh.v13i1.5169

Recebido em: 04/07/2015. Aceito em: 04/11/2015. 
15\% do número de jovens (INSTITUTO NACIONAL DE ESTATÍSTICA, 2012). Este grupo populacional não tem uma distribuição homogênea no território português. Aproximadamente $31 \%$ das pessoas idosas encontram-se na Região Norte, percentual seguindo pelos apresentados nas Regiões Centro e Lisboa com 26\%, nas regiões do Alentejo com $9,1 \%$, Algarve 4,4\%, Autônomas da Madeira $2 \%$ e dos Açores com 1,6\% (INSTITUTO NACIONAL DE ESTATÍSTICA, 2012).

Este fenômeno adquire contornos preocupantes, tanto no meio rural como urbano, devido a uma grande vulnerabilidade da pessoa idosa pelo empobrecimento. Os valores atuais das pensões de reforma ainda se situam no limiar da pobreza (DIAS, 2005). Esta condição é agravada por um sistema de proteção social recente e lacunar, pela dificuldade do sistema de saúde em responder às necessidades das pessoas idosas e pelo declínio das redes de solidariedade sociofamiliar (DIAS, 2005). A realidade descrita, acrescida da discriminação real ou simbólica e das representações sociais negativas, compromete a promoção da dignidade deste grupo populacional. Indiscutivelmente, a pessoa idosa vive e convive com inúmeros fatores que representam o desrespeito à sua individualidade, autonomia, independência, segurança e capacidade de decisão.

Nessa perspetiva, a experiência de envelhecimento pode processar-se em quadros de perversa desigualdade, injustiça e exclusão social (DIAS, 2005). Consequentemente, associado ao progressivo envelhecimento mundial da população, configura-se o fenômeno da violência contra a pessoa idosa, que ganha uma conotação especial nas sociedades impregnadas pela cultura da competitividade, da eficiência e do culto à juventude. Frequentemente silenciada pela pessoa idosa ou considerada uma forma de agir normal e naturalizada nos usos e costumes de muitas sociedades, as notificações existentes são inconclusivas sobre a magnitude deste fenômeno (MINAYO et al., 2005; MINAYO, 2005). No entanto, o progressivo aumento das expressões de violência, reproduzidas no quotidiano das relações sociais, no interior das famílias, nas instituições e em diferentes contextos, constitui-se num acontecimento desafiador no presente século.

Embora não seja um assunto muito investigado na sociedade portuguesa, este tipo de violência foi considerado um problema sério no Relatório de Prevenção contra os Maus Tratos a Idosos da Organização Mundial da Saúde (OMS) (2011). Portugal destaca-se como um dos países com maiores índices de violência $(39,4 \%)$ num universo de 53 países europeus (ORGANIZAÇÃO MUNDIAL DA SAÚDE, 2011). Este registo só é superado por quatro países: Sérvia, Áustria, Israel e Macedônia (ORGANIZAÇÃO MUNDIAL DA SAÚDE, 2011). Uma análise evolutiva efetuada pela Associação Portuguesa de Apoio à Vitima entre 2000 e 2011 assinalou que em $54 \%$ das situações, a pessoa idosa vítima de crime tinha entre os 65 e os 75 anos (ASSOCIAÇÃO PORTUGUESA DE 
APOIO À VITIMA, 2012). O sexo feminino representou a maior percentagem de pessoas idosas vítimas de crime (78\%). Relativamente ao agressor, $62,6 \%$ eram do sexo masculino, tendo $22,5 \%$ mais de 65 anos. Nesse período, a categoria de violência doméstica registar maior número de fatos criminosos (10.361; 81\%), seguindo-se a categoria de crimes contra as pessoas e a humanidade $(1.501 ; 12 \%)$ (ASSOCIAÇÃO PORTUGUESA DE APOIO À VITIMA, 2012).

Estas evidências possibilitam a formulação da seguinte questão norteadora: quais são as informações disponíveis na literatura sobre a visibilidade do fenômeno da violência contra a pessoa idosa em Portugal, no cenário familiar e institucional, tendo como contraponto os avanços alcançados em outros países? Consideramos que este tema merece uma atenção e sensibilização clínica particular por parte dos profissionais de enfermagem, na medida em que é necessário saber identificar o fenómeno da violência em contextos tão diversificados e encontrar respostas eficazes e humanizadas para os problemas que envolve. Essas respostas solicitam que o cuidado desenvolvido integre as diversas dimensões da pessoa idosa e se concretize numa relação de proximidade e de ajuda, envolvendo ações específicas de encaminhamento que visem amenizar os seus medos. Nesse sentido, este artigo tem como objetivo dar visibilidade ao fenômeno da violência contra a pessoa idosa em Portugal, com destaque para o cenário familiar e institucional. Para a sua concretização foi realizada uma revisão narrativa da literatura, considerando as suas múltiplas formas de expressão e fatores predisponentes no atual processo de envelhecimento mundial da população.

\section{Metodologia}

O presente estudo é uma revisão narrativa da literatura. Revisões narrativas são publicações amplas apropriadas para descrever e discutir o desenvolvimento ou o "estado da arte" de um determinado assunto, sob o ponto de vista teórico ou contextual (ROTHER, 2007). Não utiliza critérios explícitos e sistemáticos para a busca e análise crítica da literatura. São textos que constituem a análise da literatura científica na interpretação e análise crítica do autor (ROTHER, 2007). Embora a metodologia da revisão narrativa não permita a reprodução de dados, pode contribuir para o debate de determinadas temáticas, levantando questões e colaborando na aquisição e atualização do conhecimento em curto espaço de tempo (ROTHER, 2007).

Para o levantamento de estudos sobre esta problemática foram consultadas as seguintes bases de dados: Biblioteca Virtual em Saúde, Literatura Latino-Americana e do Caribe em Ciências da Saúde, Literatura Internacional em Ciências da Saúde, e biblioteca digital Scientific Electronic Library Online. A estratégia de busca foi baseada nos descritores em ciências da saúde: violência; pessoa idosa; maus-tratos ao idoso; violência familiar; violência institucional; enfermagem e saúde pública. Para a 
seleção dos estudos consideraram-se os seguintes critérios de inclusão: resumos completos nas bases de dados, estudos primários disponíveis na íntegra, documentos oficiais relacionados à temática da violência contra a pessoa idosa, nos idiomas inglês e português.

O processo de coleta do material foi realizado de forma não sistemática no período de Janeiro a Fevereiro de 2015, sendo encontradas 26 publicações que respondem à pergunta proposta. A partir dos artigos selecionados, foi realizada uma leitura crítica e interpretativa com imparcialidade e objetividade. A apresentação dos resultados e a discussão dos dados foram realizadas de forma descritiva, contemplando as seguintes categorias: tipologias da violência contra a pessoa idosa; evidencias na literatura; violência no cenário familiar; violência no cenário institucional.

\section{Resultados e discussão}

\section{Tipologias da violência contra a pessoa idosa}

Diversos termos são utilizados para caracterizar a violência contra a pessoa idosa: abuso, maus-tratos, negligência, omissão e abandono. Independentemente da diversidade conceitual existente neste domínio, a OMS estabelece este tipo de violência como qualquer ato, simples ou repetitivo, ou omissão de ação apropriada, que causa dano ou tensão a uma pessoa idosa e que ocorre no contexto de qualquer relacionamento em que haja uma expectativa de confiança (ORGANIZAÇÃO MUNDIAL DA SAÚDE, 2002).
Internacionalmente, foram estabelecidos alguns tipos de violência para designar as diversas ações que prejudicam a integridade física e emocional da pessoa idosa e impedem o desempenho do seu papel social (MINAYO, 2005):

- Violência Física: concerne ao uso da força física, para compelir as pessoas idosas a fazerem o que não desejam, para feri-las, provocar dor, incapacidade ou morte.

- Violência Psicológica: corresponde a agressões verbais ou gestuais com o objetivo de aterrorizar, humilhar, restringir a liberdade ou isolar do convívio social.

- Violência Sexual: refere-se ao ato ou jogo sexual de caráter homo ou heterorrelacional, utilizando pessoas idosas. Esses abusos visam obter excitação, relação sexual ou práticas eróticas por meio de aliciamento ou ameaças.

- Abandono: é uma forma de violência que se manifesta pela ausência ou deserção dos responsáveis governamentais, institucionais ou familiares de prestarem socorro a uma pessoa idosa que necessite de proteção e assistência.

- Negligência: refere-se à recusa ou à omissão de cuidados devidos e necessários à pessoa idosa por parte dos responsáveis familiares ou institucionais. São exemplos a negação de alimentos, cuidados de higiene, habitabilidade, segurança e tratamentos médicos. Esta forma de violência está frequentemente associada a outros abusos que 
geram lesões e traumas físicos, emocionais e sociais, em particular, para as pessoas idosas que se encontram em situação de múltipla dependência ou incapacidade.

- Violência Financeira ou econômica: consiste na exploração imprópria ou ilegal ou no uso não consentido pela pessoa idosa de seus recursos financeiros e patrimoniais.

- Autonegligência: diz respeito à conduta da pessoa idosa que ameaça sua própria saúde ou segurança, pela recusa de prover cuidados necessários a si mesma.

A adoção desses tipos de violência pode assegurar a comparabilidade dos resultados produzidos por diferentes estudos e a eficácia das estratégias de intervenção desenvolvidas neste domínio (DIAS, 2005).

Violência contra a pessoa idosa: evidências da literatura

Somente no final da década de 1970 e início da década de 1980, o fenômeno da violência contra a pessoa idosa no contexto familiar e institucional começou a ser reconhecido (DIAS, 2005). Os primeiros estudos surgem em $1975 \mathrm{com}$ a publicação do artigo "espancamento de avós" e com a criação de uma revista em 1989, dedicada exclusivamente ao tema - Journal of Elder Abuse \& Neglectem (DIAS, 2005). Contudo, o reconhecimento da violência sobre a mulher idosa, quer na forma de mau trato conjugal, quer na modalidade de abuso institucional foi mais tardio (DIAS, 2005). A literatura científica de países desenvolvidos e de alguns países emergentes demonstra que a violência contra a pessoa idosa é um fenômeno universal que tem como pano de fundo vários cenários (RAMOS, 2011; MINAYO, 2005; FERREIRA-ALVES, 2004; ORGANIZAÇÃO MUNDIAL DA SAÚDE, 2002). Nessa perspetiva, as situações de maus-tratos não estão confinadas aos que vivem marginalizados pela sociedade ou estão institucionalizados (FERREIRA-ALVES, 2004). Pessoas de todos os status socioeconômicos, etnias e religiões são vulneráveis como demonstram alguns estudos de várias culturas e de cunho comparativo (MINAYO, 2005; FERREIRA-ALVES, 2004).

Apesar de fazer parte do nosso quotidiano, o mundo não tem que aceitar o sofrimento e a dor que a violência provoca como parte inevitável da condição humana (ORGANIZAÇÃO MUNDIAL DA SAÚDE, 2002). Torna-se, assim, relevante reconhecer a violência como um problema de saúde pública, unificando-a como questão a ser enfrentada e definindo as distintas violências como diversidades dessa questão plural (ORGANIZAÇÃO MUNDIAL DA SAÚDE, 2002). Nesse sentido, o campo da saúde pública tem procurado liderar ações específicas que visam à definição de estratégias preventivas da violência, à promoção da qualidade de vida e de um ambiente saudável, com a superação de processos de dominação (MINAYO; SOUZA, 1999). A sociedade parece encontrar no setor da saúde um porto seguro e um 
espaço de expressão de múltiplas vozes, de inconformidades e indignações sociais (OJEDA; STRAY, 2008). Esta constatação despertou o interesse de muitos profissionais e pesquisadores no âmbito da saúde em aprofundar o conhecimento sobre a magnitude e impacto da violência, os fatores de risco e as formas de intervenção.

A violência contra a pessoa idosa manifesta-se de forma: (a) estrutural, que ocorre pela desigualdade social e é naturalizada nas manifestações de pobreza, de miséria e de discriminação; (b) interpessoal, nas formas de comunicação e de interação quotidiana; e (c) institucional, evidenciadas na aplicação ou omissão na gestão das políticas sociais pelo Estado e pelas instituições de assistência, reproduzindo relações assimétricas de poder, de domínio, de menosprezo e de discriminação (MINAYO, 2005). Múltiplas causas estão na sua origem, tendo em vista que estão implicados fatores sociais, culturais, históricos, econômicos, étnicos, familiares e individuais (RAMOS, 2011; FERREIRA-ALVES, 2004; ORTMAMN et al., 2001). Foi revelada na literatura uma associação estatística entre o abuso e alguns fatores de risco (MINAYO, 2005): idade avançada; vínculos afetivos débeis; isolamento social; reduzido nível socioeconômico; reduzido nível educacional; debilidade funcional; abuso de álcool ou drogas pelo prestador de cuidados ou pela pessoa idosa; alterações psicológicas e personalidade patológica; história de violência na família; frustração ou exaustão do prestador de cuidados; e limitação cognitiva.
Ainda que a violência contra a pessoa idosa seja objeto de estudo recente, a sua prevalência tem uma dimensão importante em todos os países em que o fenômeno foi estudado (FERREIRA-ALVES, 2004). Numa pesquisa, realizada na China, $36,2 \%$ das pessoas idosas referiram ter vivenciado pelo menos um tipo de maus-tratos (físico, emocional, negligência ou exploração financeira) no último ano (WU et al., 2012). Na Irlanda, foi identificada uma prevalência geral de $2,2 \%$ de abuso e negligência em pessoas com 65 anos ou mais, sendo o abuso financeiro e o psicológico as formas de maus-tratos mais frequentes (NAUGHTON et al., 2012). Na área rural de Mansoura, Egito, um estudo encontrou uma prevalência de $43,7 \%$ de maus-tratos por membros da família, sendo a negligência a forma mais predominante $(42,4 \%)$ (ABDEL RAHMAN; EL GAAFARY, 2012). Os autores relacionam este resultado com as diferenças culturais e condições socioeconômicas da amostra. Em Los Angeles-EUA, um estudo feito com pessoas de origem latina e idade de $66 \mathrm{ou}$ mais anos, identificou que $40,4 \%$ dessas pessoas haviam experienciado alguma forma de maus-tratos ou negligência no último ano. Aproximadamente 25\% referiram violência psicológica, $10,7 \%$ violência física, $9 \%$ abuso sexual, $16,7 \%$ exploração financeira e 11,7\% negligência por seus cuidadores (DELIEMA et al., 2012).

Percebe-se que detetar o abuso contra pessoas idosas com precisão é importante, mas igualmente difícil. Fatores 
como a subjetividade e os valores pessoais podem pesar no seu reconhecimento (FULMER et al., 2004), assim como um comportamento pode ser considerado apropriado ou normativo em uma dada cultura e ser interpretado como abusivo em outra (COHEN, 2013). Embora a OMS tenha estabelecido o conceito causas externas como indicador utilizado pelo sistema de saúde para medir a magnitude da violência, os dados registrados referem-se, exclusivamente, aos casos de lesões, traumas ou fatalidades que passam pelos serviços de saúde ou pelo Instituto Médico Legal (MINAYO, 2005). Contudo, não se pode menosprezar que estes casos conhecidos ou diagnosticados coexistem com situações invisíveis ou ainda desconhecidas da violência (MINAYO, 2005; FERREIRA-ALVES, 2004; RAMOS, 2011). Nesse sentido, os dados epidemiológicos e estatísticos são pouco consistentes e inconclusivos para dimensionar o problema, atendendo a que não consideram a elevada subnotificação deste fenômeno em todo o mundo (MINAYO et al., 2005; MINAYO, 2005). Indubitavelmente, estes dados constituem apenas a ponta do iceberg de uma cultura relacional de dominação, de conflitos intergeracionais, de negligências familiares ou institucionais (MINAYO, 2005).

A tendência da pessoa idosa para não denunciar os maus-tratos deve-se ao temor das seguintes consequências: perder o cuidador mesmo sendo este abusivo; ficar só sem ter ninguém que a cuide; ser colocada numa instituição; perder a privacidade e as relações familiares; conviver com recriminações pelo alegado abusador; expor-se publicamente e sofrer intervenção exterior; ninguém acreditar no abuso; e ser responsável pelo comportamento abusivo (FERREIRA-ALVES, 2004; FULMER et al., 2004). Estas variáveis não esgotam todas as razões da subnotificação do abuso, apenas fornecem razões plausíveis que merecem a sensibilização social, clínica e educativa dos profissionais de saúde que contactam com a pessoa idosa (FERREIRA-ALVES, 2004). Em Portugal, os estudos populacionais que estimam a magnitude desse problema são escassos (FERREIRA-ALVES, 2004; RAMOS, 2011; GONÇALVES, 2006; FERREIRA-ALVES; SOUSA, 2005). A consciência dessa realidade conduziu à realização de um estudo de caso pioneiro com o objetivo de identificar os determinantes de maus-tratos físicos, psicológicos e financeiros e da negligência contra pessoas idosas (FERREIRA-ALVES; SOUSA, 2005). Os resultados indicam a presença de indicadores de maus-tratos num número muito significativo de participantes. Os indicadores de negligência $(53,7 \%)$ e de abuso emocional $(52,4 \%)$ são os mais prevalentes, seguindo-se de forma distanciada, os indicadores de abuso financeiro $(19,5 \%) \mathrm{e}$ de abuso físico (12,2\%). O fator associado de forma considerável à negligência foi a perceção do estado de saúde. Quanto pior a perceção do estado de saúde mais indícios de abuso existem. Concluiu-se que o risco de vitimização aumenta nas pessoas com mais idade, particularmente no sexo feminino e quando o agressor 
percebe a fragilidade do estado de saúde da pessoa idosa.

Dada a sua complexidade e extensão, o conhecimento teórico produzido sobre a violência contra a pessoa idosa não é unívoco (FERREIRA-ALVES, 2004). Estudos realizados sobre esta temática apresentam diferentes metodologias e revelam diferentes prevalências (RAMOS, 2011). Obviamente, não basta descrever as ocorrências em que se cruzam necessariamente certas variáveis, interessa igualmente compreender o comportamento humano (FERREIRA-ALVES; SOUSA, 2005). A literatura demonstra que existem problemas de discriminação que subsistem e contribuem para as desigualdades de oportunidades e de poder que se manifestam sob a forma de violência desenvolvida no cenário familiar e institucional.

\section{Violência no cenário familiar}

Estudos internacionais enfatizam que a forma mais frequente de violência contra a pessoa idosa é a que ocorre no âmbito familiar (NAUGHTON, 2012; JAYAWARDENA; LIAO, 2006; LASH; PILLEMER, 2004). Frequentemente, os profissionais de saúde são as únicas pessoas que entram nas residências de pessoas idosas comprometidas (JAYAWARDENA; LIAO, 2006). Contudo, alguns profissionais sentem insegurança e dificuldade em formalizar um relato de denúncia, não sabem como proceder, ou a quem se dirigir (COHEN, 2013). A própria complexidade dos problemas de saúde na velhice também dificulta a distinção entre sinais de abuso e sintomas de várias doenças comuns nessa idade (COHEN, 2013). Esta relação de maus-tratos por membros da família é congruente com os estudos portugueses que destacam uma especial incidência para os cônjuges/companheiros (33\%), seguindo-se a relação de filho $(23,9 \%)$ (ASSOCIAÇÃO PORTUGUESA DE APOIO À VITIMA, 2012). A proporção de agressores que perpetram violência física severa e vivem com a vítima é mais elevada $(75 \%)$ do que a registrada na violência física ligeira (60\%) (RAMOS, 2011). Esses resultados permitem concluir que a família não é apenas o lugar privilegiado dos afetos e de realização pessoal, mas também do conflito, da agressão e do abuso de poder.

Parece coexistir, no interior das famílias, uma relação de amor/ódio, alicerçada numa espécie de acordo tácito que se cristaliza na violência contra pessoas mais vulneráveis. As dificuldades em detectar as situações de violência familiar aumentam em proporção direta com a idade da vítima, a existência de patologias física e/ou psicológica, com deterioração cognitiva ou com um grau de dependência que impossibilita acusar o agressor (CARREIRA, 2008). No domínio da esfera privada, a exploração do trabalho, a destituição familiar, o desenraizamento, a exploração econômica dos afetos, o abandono e a incapacidade dos cuidadores informais são apontados como situações constitutivas de violência (CARREIRA, 2008).

Particularizando a inadequada preparação dos cuidadores informais, as 
situações de violência, substancialmente graves, podem causar sofrimento tanto para quem é cuidado como para quem cuida (CARREIRA, 2008). Dessa forma, afigura-se de primordial importância a formação e o acompanhamento dos familiares face às dificuldades com que se debatem. No entanto, a sociedade portuguesa evidencia a carência de uma política de envelhecimento que favoreça a responsabilização dos familiares. Parece, assim, existir uma contradição. A família é considerada o lugar idôneo para envelhecer, mas, simultaneamente é ignorada na tomada de medidas que visem o apoio familiar para o cuidado à pessoa idosa (CARREIRA, 2008). Consequentemente, a institucionalização dos progenitores acaba por ser uma opção.

\section{Violência no cenário institucional}

A institucionalização surge como um recurso decorrente das mudanças sociais, econômicas e culturais que estão acontecendo na sociedade contemporânea e que reduzem a capacidade das famílias responderem às necessidades de atenção dos seus parentes idosos (ORGANIZAÇÃO MUNDIAL DA SAÚDE, 2002). Na sociedade portuguesa, os cuidados domiciliários ainda são percebidos como um dever que faz parte da história e experiência familiar. $\mathrm{O}$ envolvimento da família no cuidado às pessoas idosas leva a que a institucionalização em Portugal, seja uma resposta social com menos peso (DUARTE; PAÚL, 2006-2007). No entanto, dados empíricos também evidenciam que esta percentagem não é maior porque a resposta à solicitação de uma vaga é deficitária e os recursos econômicos das famílias são baixos para fazer face aos valores praticados pelas instituições (CARREIRA, 2008).

Independentemente da resposta que proporcionam, as instituições de cuidados continuados, com maior expressão, os lares e centros de acolhimento constituem um problema social e de saúde, na medida em que se transformam em lugar de segregação, estigma e preconceitos, favorecendo a ocorrência de violência (ORGANIZAÇÃO MUNDIAL DA SAÚDE, 2002; POST et al., 2010). Geralmente, essa opção não é bem acolhida pela pessoa idosa, que altera o seu quotidiano, se sente abandonada, negligenciada e desenraizada. Atendendo a que são impostas normas rígidas e atividades que homogeneízam o processo de cuidado, a individualidade de cada pessoa não é considerada, conduzindo à sua despersonalização em benefício de uma férrea orgânica normativa (CARREIRA, 2008).

Uma avaliação multidimensional do ambiente institucional de um lar público de pessoas idosas em Portugal permitiu concluir que, atendendo ao reduzido número de funcionários, o grau de apoio aos residentes era baixo (20\%), comprometendo, igualmente, a individualização do cuidado (DUARTE; PAÚL, 2006-2007). Essa instituição caracteriza-se pela insuficiente capacidade de resposta, no que se refere às expectativas de funcionamento (10\%), à política organizativa $(33 \%)$, à clareza informativa (30\%), à disponibilidade de serviços de saúde $(33 \%)$, bem como de atividades socior- 
recreativas (20\%) (DUARTE; PAÚL, 2006-2007). Para o bem-estar psicológico da pessoa idosa institucionalizada, é essencial que a mesma possa expressar a sua autossuficiência, autodeterminação e responsabilidade.

Um levantamento sobre os profissionais de instituições asilares nos Estados Unidos revelou que $36 \%$ da equipa geral relatou ter testemunhado pelo menos um incidente de abuso físico cometido por outros membros da equipa no ano anterior, enquanto $10 \%$ admitiram ter cometido, eles próprios, pelo menos um ato de abuso físico (ORGANIZAÇÃO MUNDIAL DA SAÚDE, 2002). Essas descobertas sugerem que o número de maus-tratos contra pessoas idosas residentes em instituições pode ser muito mais extenso do que geralmente se acredita. Algumas mortes, tanto em cenários institucionais como na comunidade, têm sido frequentemente atribuídas a causas naturais, acidentais ou indeterminadas, quando na verdade foram consequências de comportamento abusivo ou negligente (ORGANIZAÇÃO MUNDIAL DA SAÚ$\mathrm{DE}, 2002)$.

$\mathrm{O}$ conhecimento da violência institucional raramente chega às fontes de informação oficiais, ficando limitada à denúncia informal. No entanto, é preciso sensibilizar para o fato de em instituições médicas e sociais, criadas para cuidar e proteger as pessoas idosas, podem ocorrer situações de violência de forma invisível (ORGANIZAÇÃO MUNDIAL DA SAÚDE, 2002). Exemplificando, a aceitação ou a negação de que a violência faz parte do quotidiano dos profissionais de saúde no contexto hospitalar depende das diferentes perceções, do tipo de conhecimento sobre o fenômeno e da "cultura do silêncio" (SOUZA; MEIRA; MENEZES, 2012). Alguns profissionais de saúde identificam certas práticas como formas de maus-tratos e negligência, em contexto familiar, mas não as consideram atos violentos ou abusivos em contexto profissional, fazendo até parte dos normativos e procedimentos profissionais (GIL; FERNANDES, 2011).

Este ponto de vista contribui para a legitimação de interesses e posições profissionais nas organizações com repercussões na identificação das situações de violência (GIL; FERNANDES, 2011). Outros profissionais guardam no imaginário a representação da violência como uma ação que produz sinais corporais visíveis ameaçadores da vida. No entanto, a violência institucional não é menos brutal que a violência física, pois deflagra sentimentos de culpa, solidão, dependência, inutilidade e aumenta a sensação de desamparo (SOUZA; MEIRA; MENEZES, 2012). Pode-se assim apreender que as diversas ações são distintamente interpretadas por quem as exerce, por quem as sofre e por quem as observa, diagnostica, avalia e propõe sugestões de intervenção (CARREIRA, 2008). Identificada nos espaços públicos e privados, nas relações institucionais, grupais ou interpessoais, não existem elementos suficientes para lidar com um domínio tão amplo como a violência, alimentado por interconexões ainda pouco conhecidas (SCHRAIBER; D'OLIVEIRA; COUTO, 2006). Diante dessa realidade, 
aprofundar o conhecimento rigoroso sobre este domínio constitui uma das principais prioridades mundiais (ORGANIZAÇÃO MUNDIAL DA SAÚDE, 2002). De um modo paradoxal, vivenciamos na contemporaneidade, a expansão tanto de domínios da violência, como dos direitos humanos e sociais (SCHRAIBER; D'OLIVEIRA; COUTO, 2006).

\section{Conclusão}

Apesar de incipientes, as evidências da literatura sobre a problemática da violência contra a pessoa idosa na sociedade portuguesa são corroboradas pelos dados encontrados na literatura internacional. Uma compreensão mais aprofundada do processo de construção da violência no ambiente familiar e institucional permitirá resignificar um problema complexo, histórico e dinâmico, que está disseminado por todos os estratos sociais. Essa evidência justifica o destaque que tem merecido no campo da saúde, no sentido de capacitar profissionais de saúde para a identificação das situações de risco e desenvolvimento de intervenções que permitam prevenir ou atenuar os seus efeitos para a pessoa idosa, seus cuidadores e seus familiares. O número de pessoas idosas vem aumentando na sociedade portuguesa, mas o seu estatuto social contínua diminuído. $\mathrm{O}$ acentuado grau de dependência, isolamento social e fragilidade econômica conduzem a uma maior vulnerabilidade e risco de incidência de violência. Consequentemente, salvaguardar os direitos das pessoas idosas é uma matéria que diz respeito a todos os segmentos da sociedade em geral. Torna-se, assim, imperioso fomentar a solidariedade entre gerações e desenvolver uma consciência social integradora, que reconheça o seu valor e contemple a igualdade de oportunidades. Essa valorização será determinante na procura de soluções interdisciplinares que visem preservar a integridade, a dignidade, as capacidades e as experiências de vida da pessoa idosa. Esse é um caminho que pode ser trilhado pelos profissionais envolvidos no cuidado às pessoas idosas visando à transformação da realidade em que vivemos.

\section{Violence against the elderly in the Portuguese context: issues and contradictions}

\section{Abstract}

The violence against elderly people is a universal phenomenon with several scenarios as a backdrop. In a universe of 53 European countries, Portugal stands out with one of the highest rates $(39.4 \%)$. Considering its high underreporting, the epidemiological data are only the tip of the iceberg of a relational culture of domination, intergenerational conflict or of family or institutional negligence. This article aims to give visibility to the phenomenon of violence against the elderly in Portugal considering its multiple forms of expression and predisposing factors in the current world population ageing process. In this sense, a narrative review was carried out to support this reflection in the Portuguese context, having as a counterpoint the advances made in other countries. It is concluded that the recognition of this type of violence as a serious problem in Portugal requires a 
social and integrative consciousness, and an effort of complementarity of the various areas of knowledge. Only in this way will it be possible to safeguard the rights of the old people and find effective and humanized responses to prevent or mitigate the effects of violence in society.

Keywords: Old people. Public health. Violence.

\section{Referências}

ABDEL RAHMAN, T. T.; EL GAAFARY, M. M. Elder mistreatment in a rural area in Egypt. Geriatrics Gerontology Internacional, v. 12 , n. 3, p. 532-537, 2012.

ASSOCIAÇÃO PORTUGUESA DE APOIO À VITIMA. Pessoas idosas vítimas de crime e de violência 2000/2011. Lisboa: Estatísticas APAV, 2012. Disponível em: <http:// www.apav.pt/apav_v2/images/pdf/Estatisticas_APAV_Pessoas_Idosas_2000-2011.pdf>. Acesso em: 10 jan. 2015.

CARREIRA, J. Estudo sobre as medidas de intervenção social nos maus tratos ao idoso. Dissertação (Mestrado em Trabalho Social) - Faculdade de Ciências Humanas e Sociais, Universidade Fernando Pessoa, Porto, 2008.

COHEN, M. The process of validation of a three-dimensional model for the identification of abuse in older adults. Archives of Gerontology and Geriatrics, v. 57, n. 3, p. 243-249, 2013.

DIAS, I. Envelhecimento e violência contra os idosos. Sociologia. Revista da Faculdade de Letras do Porto, n. 15, p. 249-273, 2005.

DELIEMA, M. et al. Determining prevalence and correlates of elder abuse using promotores: low-income immigrant Latinos report high rates of abuse and neglect. Journal of the American Geriatrics Society, v. 60, n. 7, p. 1.333-1.339, jul. 2012.

DUARTE, M.; PAÚL, C. Avaliação do ambiente institucional - público e privado: estudo comportamental dos idosos. Revista Transdisciplinar de Gerontologia, v. 1, n. 1, p. 17-29, 2006-2007.

FERREIRA-ALVES, J. Fatores de risco e indicadores de abuso e negligência de idosos. Repositório da Universidade do Minho, 2004. Disponível em: <http://repositorium.sdum. uminho.pt/handle/1822/4423>. Acesso em: 10 jan. 2015.

FERREIRA-ALVES, J.; SOUSA, M. Indicadores de maus tratos em pessoas idosas na cidade de Braga: estudo preliminar. Revista Sociologia da Faculdade de Letras da Universidade do Porto, n. 15, p. 303-313, 2005.

FULMER, T. et al. Progress in elder abuse screening and assessment instruments. Journal of the American Geriatrics Society, v. 52, n. 2, p. 297-304, 2004.

GIL, A. P.; FERNANDES, A. A. "No trilho da negligência..." configurações exploratórias de violência contra pessoas idosas. Fórum Sociológico, Lisboa, n. 21, 2011. Disponível em: $<$ http://sociologico.revues.org/471>. Acesso em: 15 jan. 2015.

GONÇALVES, C. A. Idosos: abuso e violência. Dossier Saúde dos Idosos. Revista Portuguesa de Clinica Geral, n. 22, p. 739-745, 2006.

INSTITUTO NACIONAL DE ESTATÍSTICA. Destaque: Censos 2011 - Resultados pré-definitivos. Lisboa: Instituto Nacional de Estatística, 2012.

JAYAWARDENA, K. M.; LIAO, S. Elder abuse at end of life. Journal of Palliative Medicine, v. 9, n. 1, p. 127-136, 2006.

LASH, M. S.; PILLEMER, K. Elder abuse. Lancet, n. 364, p. 1.263-1.272, 2004.

MINAYO, M. C. S. Violência contra idosos: o avesso do respeito à experiência e à sabedoria. 2. ed. Brasília: Secretaria Especial dos Direitos Humanos, 2005.

MINAYO, M. C. S.; SOUZA, E. R. É possível prevenir a violência? Reflexões a partir do 
campo da saúde pública. Ciência \& Saúde Coletiva, v. 4, n. 1, p. 7-32, 1999.

MINAYO, C. et al. Plano de Ação para o Enfrentamento da Violência contra a Pessoa Idosa. Brasília: Subsecretaria de Direitos Humanos, 2005.

NAUGHTON, C. et al. Elder abuse and neglect in Ireland: results from a national prevalence survey. Age and Ageing, v. 41, n. 1, p. 98-103, 2012. Disponível em: <http:// ageing.oxfordjournals.org/content/41/1/98>. Acesso em: 20 fev. 2015.

OJEDA, B. S.; STRAY, M. N. Saberes e poderes em saúde: um olhar sobre as relações interprofissionais. Revista Ciência \& Saúde, Porto Alegre, v. 1, n. 1, p. 2-8, 2008.

ORGANIZAÇÃO MUNDIAL DA SAÚDE. European report on preventing elder maltreatment. 2011. Disponível em: <http://www. euro.who.int/en/what-we-publish/abstracts/ european-report-on-preventing-elder-maltreatment>. Acesso em: 15 fev. 2015.

. Relatório mundial sobre violência e saúde. Genebra, 2002.

ORTMAMN, C. et al. Fatal neglect of the elderly. Journal of Legal Medicine, n. 114, p. 191-193, 2001.

POST, L. et al. Elder abuse in long-term care: types, patterns, and risk factors. Research on Aging, v. 32, n. 3, p. 323-348, 2010.

RAMOS, F. da S. Os agressores de pessoas idosas. Dissertação (Mestrado em Educação para a Saúde) - Faculdade de Psicologia e Ciências da Educação, Universidade do Porto, Porto, 2011.

ROTHER, E. T. Revisão sistemática x revisão narrativa. Acta Paulista de Enfermagem, São Paulo, v. 20, n. 2, p. v-vi, 2007.

SCHRAIBER, L. B.; D'OLIVEIRA, A. F. P. L.; COUTO, M. T. Violência e saúde: estudos científicos recentes. Revista Saúde Pública, São Paulo, n. 40 (especial), p. 112-120, 2006.
SOUZA, A.; MEIRA, E.; MENEZES, M. R. Violência contra as pessoas idosas promovida em instituição de saúde. Mediações - Revista de Ciências Sociais, Londrina, v. 17, n. 2, p. 57-72, 2012.

WU, L. et al. Prevalence and associated factors of elder mistreatment in a rural community in People's Republic of China: a cross-sectional study. PLoS One, v. 7, n. 3, p. e33.857, 2012. 